\title{
THE DISCRETE AND CONTINUOUS PAINLEVÉ VI HIERARCHY AND THE GARNIER SYSTEMS
}

\author{
F. W. NIJHOFF and A. J. WALKER \\ Department of Applied Mathematics, The University of Leeds, Leeds LS2 9JT, UK \\ e-mail:frank@amsta.leeds.ac.uk andamtajw@amsta.leeds.ac.uk
}

(Received 2 January, 2000; revised 5 October, 2000)

\begin{abstract}
We present a general scheme to derive higher-order members of the Painlevé VI $\left(\mathrm{P}_{\mathrm{VI}}\right)$ hierarchy of ODE's as well as their difference analogues. The derivation is based on a discrete structure that sits on the background of the $\mathrm{P}_{\mathrm{VI}}$ equation and that consists of a system of partial difference equations on a multidimensional lattice. The connection with the isomonodromic Garnier systems is discussed.
\end{abstract}

1991 Mathematics Subject Classification. 34E99, 33E30, 58F07, 39A10.

1. Introduction. In recent years there has been a growing interest in discrete analogues of the famous Painlevé equations; i.e. nonlinear nonautonomous ordinary difference equations tending to the continuous Painlevé equations in a well-defined limit and which are integrable in their own right; cf. [1]. Even though the qualitative features of the solutions of these systems are not yet fully understood, nonetheless in most of the known examples the main ingredients of their integrability have been exhibited. Recently, a classification of continuous as well as discrete Painlevé equations, in terms of the root systems associated with affine Weyl groups, has been proposed on the basis of the singularities of the rational surfaces of their initial conditions and their blowings-up; cf. [2].

In a recent paper [3] we established a connection between the continuous Painlevé VI $\left(\mathrm{P}_{\mathrm{VI}}\right)$ equation and a non-autonomous ordinary difference equation depending on four arbitrary parameters. This novel example of a discrete Painlevé equation arises on the one hand as the nonlinear addition formula for the $\mathrm{P}_{\mathrm{VI}}$ transcendents, in fact what is effectively a superposition formula for its BäcklundSchlesinger transforms, on the other hand from the similarity reduction on the lattice (cf. $[4,5]$ ), of a system of partial difference equations associated with the lattice $\mathrm{KdV}$ family. In subsequent papers [6,7] some more results on these systems were established, namely the existence of the Miura chain and the discovery of a novel Schwarzian PDE generating the entire (Schwarzian) KdV hierarchy of nonlinear evolution equations and whose similarity reduction is exactly the $\mathrm{P}_{\mathrm{VI}}$ equation, this being to our knowledge the first example of an integrable scalar PDE that reduces to full $\mathrm{P}_{\mathrm{VI}}$ with arbitrary parameters.

In the present note we extend these results to multidimensional systems associated with higher-order generalisations of the $\mathrm{P}_{\mathrm{VI}}$ equation. Already in [3] we noted that the similarity reduction of the lattice $\mathrm{KdV}$ system could be generalised in a natural way to higher-order differential and difference equations without, however, clarifying in detail the nature of such equations. We shall argue here that, in fact, such equations constitute what one could call the Painlevé VI hierarchy and its discrete counterpart. Whilst the idea of constructing hierarchies of Painlevé equations 
by exploiting the similarity reductions of hierarchies of nonlinear evolution equations of $\mathrm{KdV}$ type is at least two decades old, cf. [8], the issue has gained renewed interest in recent years, cf. e.g. [9-13], because of the hypothetical possibility that these hierarchies of higher-order Painlevé equations yield new transcendents. Evidence to that effect might be given by the asymptotic analysis of the higher-order equations, since they seem to be governed by hyper-elliptic functions rather than elliptic ones, as is the case for the original Painleve equations [14].

Most of the existing results on hierarchies of discrete and continuous Painlevé equations are restricted to the examples of $\mathrm{P}_{\mathrm{I}}$ and $\mathrm{P}_{\mathrm{II}}$ hierarchies, since only in these cases is it clear which hierarchies of nonlinear evolution equations should be taken as the starting point for their construction. In the case of the other Painleve equations, notably $\mathrm{P}_{\mathrm{VI}}$, it has been less clear what to take as a starting point for the construction of its hierarchy. With the results of $[3,6,7]$ we are now well-equipped to tackle this problem, and in the present paper we outline the basic construction of the equations in the discrete as well as the continuous $\mathrm{P}_{\mathrm{VI}}$ hierarchy. In fact, we shall demonstrate that the lattice $\mathrm{KdV}$ system can be naturally embedded in a multidimensional lattice system achieving the higher-order reductions by including more terms in the relevant similarity constraint that provokes the coupling between the various lattice directions.

It should be noted that in a sense higher-order $\mathrm{P}_{\mathrm{VI}}$ systems already were constructed by R. Garnier in his celebrated paper [15] of 1912, extending the original approach of $\mathrm{R}$. Fuchs who was the first in [16] to find $\mathrm{P}_{\mathrm{VI}}$ arising from the isomonodromic deformation of a second-order linear differential equation. We shall conclude our paper with a discussion of these Garnier systems, which in view of the recent interest in algebraic solutions of $\mathrm{P}_{\mathrm{VI}}$, cf. e.g. [17-20], deserve in our opinion some renewed attention.

2. The discrete PVI hierarchy. In [3], following earlier work e.g. [4,5], (cf. also [1]), a coherent framework was developed in which the similarity reduction of both discrete as well as continuous equations associated with the lattice $\mathrm{KdV}$ family were treated. Surprisingly, from these reductions the full $\mathrm{P}_{\mathrm{VI}}$ equation for arbitrary parameters emerged together with a four-parameter discrete equation, i.e. a discrete Painlevé equation. From the treatment of [3] it was evident how to extend the lattice system of partial difference equations and their similarity constraints leading to the reductions to $\mathrm{P}_{\mathrm{VI}}$. Here we describe explicitly this higher-dimensional lattice system and discuss their explicit reductions.

The lattice $\mathrm{KdV}$ family of equations contains many related equations such as the lattice Schwarzian $\mathrm{KdV}$, the lattice modified $\mathrm{KdV}(\mathrm{mKdV})$ and the actual lattice $\mathrm{KdV}$ equations. We concentrate here on one member of this family only, namely the lattice $\mathrm{mKdV}$ equation:

$$
p v_{n, m} v_{n, m+1}+q v_{n, m+1} v_{n+1, m+1}=q v_{n, m} v_{n+1, m}+p v_{n+1, m} v_{n+1, m+1},
$$

(cf. e.g. [21]), with discrete independent variables $n, m$ and depending on additional parameters of the equation $p, q$, i.e. the lattice parameters. As was pointed out earlier, cf. [21], the lattice equation (2.1) actually represents a compatible parameterfamily of partial difference equations: namely, we can embed the equation (2.1) into a multidimensional lattice by imposing a copy of (2.1) with different parameters on 
any two-dimensional sublattice, identifying each lattice direction with a corresponding lattice parameter $p_{i} \in \mathbb{C}$ in which direction the sites are labelled by discrete variables $n_{i}$ (noting that these are not necessarily integers, but shift by units, i.e. $\left.n_{i} \in \theta_{i}+\mathbb{Z}, \theta_{i} \in \mathbb{C}\right)$. Thus, combining two different lattice directions, labelled by $(i, j)$ we can write the lattice equation (2.1) on the corresponding sublattice as

$$
p_{i} v v^{j}+p_{j} v^{j} v^{i j}=p_{j} v v^{i}+p_{i} v^{i} v^{i j}
$$

in which we use the right superscripts $i, j$ to denote the shifts in the corresponding directions, whereas we use left subscripts $i, j$ to denote shifts in the reverse direction; i.e.

$$
v=v(\mathbf{n} ; \mathbf{p}), \quad v^{j}=T_{j} v(\mathbf{n} ; \mathbf{p})=v\left(\mathbf{n}+\mathbf{e}_{j} ; \mathbf{p}\right), \quad{ }_{j} v=T_{j}^{-1} v(\mathbf{n} ; \mathbf{p})=v\left(\mathbf{n}-\mathbf{e}_{j} ; \mathbf{p}\right),
$$

where $\mathbf{n}$ denotes the vector of the discrete variables $n_{i}$, for all lattice directions labelled by $i$, each corresponding to the component $p_{i}$ of the vector $\mathbf{p}$ of lattice parameters. We use the symbol $\mathbf{e}_{j}$ to denote the vector with single nonzero entry equal to unity in its $j^{\text {th }}$ component.

The consistency of the lattice equation (2.2) along the multidimensional lattice follows from Figure 1: considering the three-dimensional sublattice with elementary directions $\left\{\mathbf{e}_{1}, \mathbf{e}_{2}, \mathbf{e}_{3}\right\}$ then on each elementary cube in this lattice the iteration of initial data proceeds along the six faces of this cube, on each of which we have an equation of the form (2.2). Thus, starting from initial data $v, v^{1}, v^{2}, v^{3}$ we can then uniquely calculate the values of $v^{12}, v^{13}$ and $v^{23}$ by using the equation. However, proceeding further there are in principle three different ways to calculate the value of $v^{123}$, unless the equation satisfies (as is the case for the equation (2.2)) the special property that these three different ways of calculating this point actually lead to one and the same value. It is indeed at this point that the consistency of the embedding of the lattice $\mathrm{MKdV}$ into the multidimensional lattice is tested. In fact, by using equation (2.2) to eliminate all terms shifted in two directions in favour of terms shifted in only one direction we find that this value is given by

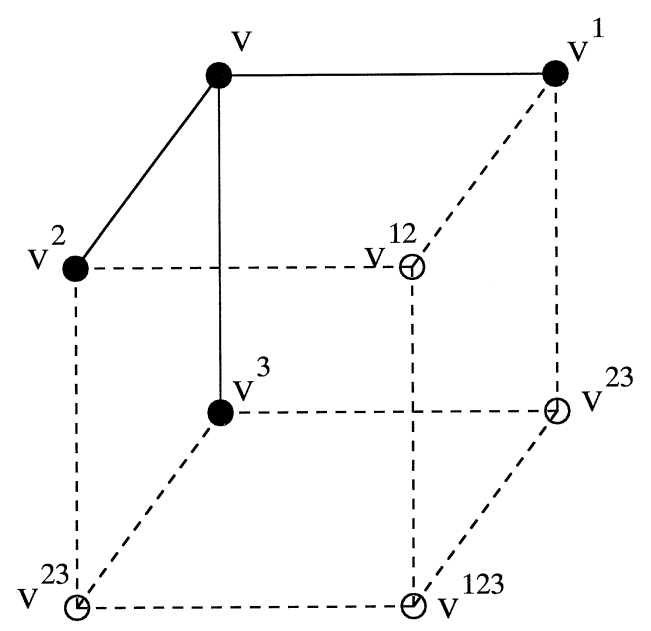

Figure 1. Consistency of the lattice equation. 


$$
\begin{aligned}
v^{i j k}= & \frac{\left(p_{i}-p_{k}\right)\left(p_{i}+p_{k}\right) p_{j} v^{i} v^{k}+\left(p_{j}-p_{i}\right)\left(p_{j}+p_{i}\right) p_{k} v^{j} v^{i}+\left(p_{k}-p_{j}\right)\left(p_{k}+p_{j}\right) p_{i} v^{j} v^{k}}{\left(p_{i}-p_{k}\right)\left(p_{i}+p_{k}\right) p_{j} v^{j}+\left(p_{j}-p_{i}\right)\left(p_{j}+p_{i}\right) p_{k} v^{k}+\left(p_{k}-p_{j}\right)\left(p_{k}+p_{j}\right) p_{i} v^{i}} \\
& (i, j, k=1,2,3),
\end{aligned}
$$

(which is clearly invariant for any permutation of the labels ijk), independent of the way in which we calculate this value! Thus, the equation (2.2) can be simultaneously imposed on functions $v\left(n_{1}, n_{2}, n_{3}, \ldots\right)$ of the lattice sites. This is precisely the discrete analogue of the hierarchy of commuting higher-order flows of the (modified) $\mathrm{KdV}$ equation!

As a consequence of this compatibility we shall call the system (2.2) a holonomic system of partial difference equations.

The continuous equation for the $\mathrm{P}_{\mathrm{VI}}$ hierarchy follows from the differential equations with respect to the lattice parameters $p_{i}$, also given in [3], which read

$$
-p_{i} \frac{\partial}{\partial p_{i}} \log v=n_{i} a_{i}
$$

in which the variable $a_{i}$ is given by

$$
a_{i} \equiv \frac{v^{i}-{ }_{i} v}{v^{i}+{ }_{i} v} .
$$

It can be shown that the differential relations (2.3) are actually compatible not only amongst themselves, but also with the the discrete equations on the lattice (2.2); i.e. the discrete and continuous flows are commuting:

$$
\frac{\partial}{\partial p_{i}}\left(\frac{\partial v}{\partial p_{j}}\right)=\frac{\partial}{\partial p_{j}}\left(\frac{\partial v}{\partial p_{i}}\right), \quad \frac{\partial v^{i}}{\partial p_{j}}=T_{i}\left(\frac{\partial v}{\partial p_{j}}\right) .
$$

This can actually be demonstrated by explicit calculation exploiting the discrete relations (2.10) below, but we shall not give the details here (which follow closely the pattern of calculations of [3]). Thus, we have here a large multidimensional system of equations with discrete (in terms of the variables $n_{i}$ ) as well as continuous (in terms of the parameters $p_{i}$ ) commuting flows, in terms of which compatible equations of three different types (partial difference, differential-difference and partial differential) figure in one and the same framework: the partial difference equations are precisely the lattice equations (2.2), the differential-difference equations are the relations (2.3), whilst for the partial differential equations in the scheme we refer to our recent paper [7].

Now, we turn to the issue of the symmetry reduction of the multidimensional lattice in the sense of [5]. It follows from the general framework of [3] that the similarity constraint for the multidimensional lattice $\mathrm{MKdV}$ system is as follows:

$$
\sum_{i} n_{i} a_{i}=\mu-v, \quad v=\lambda(-1)^{\sum_{i} n_{i}}
$$

$\mu$ and $\lambda$ being constants. The sum in (2.5) is over all the $i$ labelling the lattice directions, the choice of which decides the order of the reduction. 
To analyse the reduction it is convenient to introduce the following variables:

$$
\begin{gathered}
x_{i j} \equiv \frac{v}{v^{i j}} \quad \Rightarrow \quad{ }_{i} x_{i j} \equiv T_{i}^{-1} x_{i j}=\frac{{ }_{i} v}{v^{j}}, \\
X_{i j} \equiv \frac{v^{i}}{v^{j}} \quad \Rightarrow \quad{ }_{i} X_{i j} \equiv T_{i}^{-1} X_{i j}=\frac{v}{{ }_{i} v^{j}} .
\end{gathered}
$$

The variables $x_{i j}=x_{j i}$ and $X_{i j}=1 / X_{j i}$ are not independent, but related via

$$
X_{i j}=\frac{p_{i} x_{i j}+p_{j}}{p_{j} x_{i j}+p_{i}} \Leftrightarrow x_{i j}=\frac{-p_{i} X_{i j}+p_{j}}{p_{j} X_{i j}-p_{i}},
$$

which follow directly from the definitions and (2.2). Furthermore, we have

$$
\frac{T_{i}^{-1} x_{i j}}{X_{i j}}=\frac{{ }_{i} v}{v^{i}}=\frac{1-a_{i}}{1+a_{i}}
$$

which follows from the definitions of $a_{i},(2.4)$ and those of $x_{i j}$ and $X_{i j},(2.6)$ and (2.7). In order to obtain explicit equations from the reduction given by the constraint (2.5) we need a number of relations for the objects $a_{i}$ which follow from (2.2), namely

$$
1+a_{j}^{i}=\frac{\left(p_{i} X_{i j}-p_{j}\right)\left(a_{j}+1\right)+2 p_{j}}{p_{i} x_{i j}+p_{j}} \quad(i \neq j),
$$

which expresses shifted $a_{j}$ in terms of unshifted objects and, from the definition together with (2.9), we obtain

$$
\begin{aligned}
a_{i} & =\frac{p_{j i} X_{i j} X_{i j}+p_{i}\left({ }_{i} X_{i j}-X_{i j}\right)-p_{j}}{p_{j i} X_{i j} X_{i j}-p_{i}\left({ }_{i} X_{i j}+X_{i j}\right)+p_{j}} \\
& =\frac{-p_{j i} x_{i j} x_{i j}-p_{i}\left({ }_{i} x_{i j}-x_{i j}\right)+p_{j}}{p_{j i} x_{i j} x_{i j}+p_{i}\left({ }_{i} x_{i j}+x_{i j}\right)+p_{j}} \quad(i \neq j),
\end{aligned}
$$

which expresses $a_{i}$ in terms of shifted $x_{i j}$ or $X_{i j}$.

First we shall focus now on the reductions under the symmetry constraint (2.5) to derive closed-form ordinary differential equations (ODE's) choosing one particular lattice parameter $p_{i}$ as our independent variable. To implement this reduction explicitly we first need to derive differential relations for the $a_{j}$. By using (2.3) in combination with (2.4) we easily obtain

$$
-p_{i} \frac{\partial}{\partial p_{i}} \log \left(\frac{1-a_{j}}{1+a_{j}}\right)=n_{i}\left({ }_{j} a_{i}-a_{i}^{j}\right),
$$

which, on using (2.10), in combination with (2.6)-(2.9), to eliminate the forward and backward shifted $a_{i}$, yields after a lengthy calculation the following differential relation:

$$
\frac{\partial a_{j}}{\partial p_{i}}=\frac{n_{i} p_{j}}{\left(p_{j}-p_{i}\right)\left(p_{j}+p_{i}\right)}\left[\left(1+a_{i}\right)\left(1-a_{j}\right) X_{j i}-\left(1+a_{j}\right)\left(1-a_{i}\right) X_{i j}\right] \quad(i \neq j) .
$$


A similar calculation yields the following relations for the reduced variables $X_{i j}$

$$
\begin{aligned}
\mu+v+p_{i} \frac{\partial}{\partial p_{i}} \log X_{i j}= & n_{i} \mathcal{X}_{j i} a_{i}+\sum_{k \neq i} n_{k} \mathcal{X}_{i k} a_{k}+n_{i} \frac{p_{i} p_{j}}{\left(p_{i}-p_{j}\right)\left(p_{i}+p_{j}\right)}\left(X_{j i}-X_{i j}\right) \\
& +\sum_{k \neq i} n_{k} \frac{p_{k} p_{i}}{\left(p_{k}-p_{i}\right)\left(p_{k}+p_{i}\right)}\left(X_{i k}-X_{k i}\right),
\end{aligned}
$$

in which we have abbreviated

$$
\mathcal{X}_{i j} \equiv \frac{\left(p_{i} X_{i j}-p_{j}\right)\left(p_{j}-p_{i} X_{j i}\right)}{\left(p_{j}-p_{i}\right)\left(p_{j}+p_{i}\right)}=-\mathcal{X}_{j i}
$$

Using (2.13) in conjunction with (2.14) and using the similarity constraint (2.5) to eliminate the $a_{i}$, we obtain a coupled first-order system of ODE's w.r.t. the independent variable $t_{i}=p_{i}^{2}$ in terms of the $2 N-2$ variables $a_{k}, X_{i k},(k \neq i)$. Solving the variables $a_{k}$ from the linear system given by the equations (2.14) and inserting them into (2.13), we obtain a coupled set of second-order nonlinear ODE's for the variables $X_{i k}$. It is this set of equations that makes up the continuous PVI hierarchy.

Second, turning our attention to the discrete case, we note that since the lefthand side of (2.11) depends only on the label $i$ but not on $j$, for fixed $i$ this represents a set of $N-2$ coupled first-order ordinary difference equations ( $\mathrm{O} \Delta \mathrm{E}$ 's) with respect to the shift in the discrete variable $n_{i}$ between the $N-1$ variables $X_{i j},(j \neq i)$. Furthermore, the relations (2.10), for the same fixed label $i$, provide us with a set of $N-1$ first-order relations between the variables $a_{j},(j \neq i)$, and thus together with the similarity constraint (2.5), where $a_{i}$ is substituted by (2.11), we obtain a set of $2(N-1)$ first-order nonlinear $\mathrm{O} \Delta \mathrm{E}$ 's for the $2(N-1)$ variables $X_{i j}, a_{j},(j \neq i)$, which together form our higher-order discrete system.

3. Special cases: $\boldsymbol{N}=\mathbf{2}, \boldsymbol{N}=\mathbf{3}$. In the previous section we described how the symmetry constraint (2.5) could be used to reduce the partial difference equations (2.2) and the differential-difference equations (2.3) to coupled systems of ODE's (in the continuous case) and O $\Delta$ E's (in the discrete case). We shall now give explicit examples of these coupled systems in the particular cases $N=2$ and $N=3$.

$\boldsymbol{N}=\mathbf{2}$. We shall be very brief about the two-dimensional case $N=2$ that was the main subject of study in the earlier paper [3]. There it was shown how in a consistent way an initial value problem (IVP) can be formulated, employing both the similarity constraint and the lattice equation, and how this system can be explicitly reduced using the relations exhibited in Section 2. Using in this case the slightly more convenient notation

$$
a_{1}=a, \quad a_{2}=b, \quad x_{12}=x, \quad X_{12}=X
$$

and using the discrete independent variables $n_{1}=n, n_{2}=m$, as well as the lattice parameters $p_{1}=p, p_{2}=q$, we derived the second order nonlinear non-autonomous difference equation 


$$
\begin{aligned}
\frac{2(n+1)}{1-y_{n+1} y_{n}} & +\frac{2 n}{1-y_{n} y_{n-1}}=\mu+\lambda(-1)^{n}+2 n+1+ \\
& +\frac{\left(\mu-\lambda(-1)^{n}\right)\left(r^{2}-1\right) y_{n}+r\left(1-y_{n}^{2}\right)\left[\left(n+\frac{1}{2}\right)-\left(m+\frac{1}{2}\right)(-1)^{n}\right]}{\left(r+y_{n}\right)\left(1+r y_{n}\right)}
\end{aligned}
$$

(the notation deviates slightly from the one of [3]), where $r=p / q$ and where the variables $y_{n}$ are related to the $X$ and $x$ by the prescription: $y_{2 n}=x(2 n)$ for the even sites, whilst $y_{2 n+1}=-1 / X(2 n+1)$ for the odd lattice sites (the latter choice being mainly motivated by the wish to cast the equation into a convenient shape). It was pointed out in [3] that whilst a continuum limit of (3.1) yields the $\mathrm{P}_{\mathrm{V}}$ equation, its general solution can be expressed in terms of $\mathrm{P}_{\mathrm{VI}}$ transcendents (noting its dependence on four arbitrary parameters, $\mu, \lambda, r$ and $m$ ).

The continuous equation for the variable $X$ in terms of the lattice parameter $p$ as independent variable in this case reads

$$
\begin{aligned}
& p\left(p^{2}-q^{2}\right)^{2} X(q X-p)(p X-q) \frac{\partial^{2} X}{\partial p^{2}}= \\
& =\frac{1}{2} p\left(p^{2}-q^{2}\right)^{2}\left[p q\left(3 X^{2}+1\right)-2\left(p^{2}+q^{2}\right) X\right]\left(\frac{\partial X}{\partial p}\right)^{2}+ \\
& +\left(q^{2}-p^{2}\right)\left[2 p^{2} X(p X-q)(q X-p)+\left(q^{2}-p^{2}\right)^{2} X^{2}\right] \frac{\partial X}{\partial p} \\
& +\frac{1}{2} q\left[\left(\alpha X^{2}-\beta\right)(p X-q)^{2}(q X-p)^{2}+\left(p^{2}-q^{2}\right) X^{2}\left((\gamma-1)(q X-p)^{2}-(\delta-1)(p X-q)^{2}\right)\right],
\end{aligned}
$$

and it is not difficult to show that this is actually the $\mathrm{P}_{\mathrm{VI}}$ equation through the identification $w(t)=p X(p)$, where $t=p^{2}$, and setting $q=1$, leading to

$$
\begin{aligned}
\frac{d^{2} w}{d t^{2}}= & \frac{1}{2}\left(\frac{1}{w}+\frac{1}{w-1}+\frac{1}{w-t}\right)\left(\frac{d w}{d t}\right)^{2}-\left(\frac{1}{t}+\frac{1}{t-1}+\frac{1}{w-t}\right) \frac{d w}{d t} \\
& +\frac{w(w-1)(w-t)}{8 t^{2}(t-1)^{2}}\left(\alpha-\beta \frac{t}{w^{2}}+\gamma \frac{t-1}{(w-1)^{2}}-(\delta-4) \frac{t(t-1)}{(w-t)^{2}}\right)
\end{aligned}
$$

with the identification of the parameters $\alpha, \beta, \gamma, \delta$ as follows:

$$
\begin{array}{ll}
\alpha=(\mu-v+m-n)^{2}, & \beta=(\mu-v-m+n)^{2}, \\
\gamma=(\mu+v-m-n-1)^{2}, & \delta=(\mu+v+m+n+1)^{2} .
\end{array}
$$

Equation (3.2) is interesting in its own right since it provides us with a covariant way of writing $\mathrm{P}_{\mathrm{VI}}$, noting its invariance under the transformations:

$$
n \leftrightarrow m, \quad p \leftrightarrow q, \quad X \leftrightarrow 1 / X .
$$

$N=3$. This first higher-order case deals with the first genuinely multidimensional situation of three two-dimensional sublattices, on each of which a copy of the lattice MKdV equation (2.2) is defined. In addition there is also the similarity 

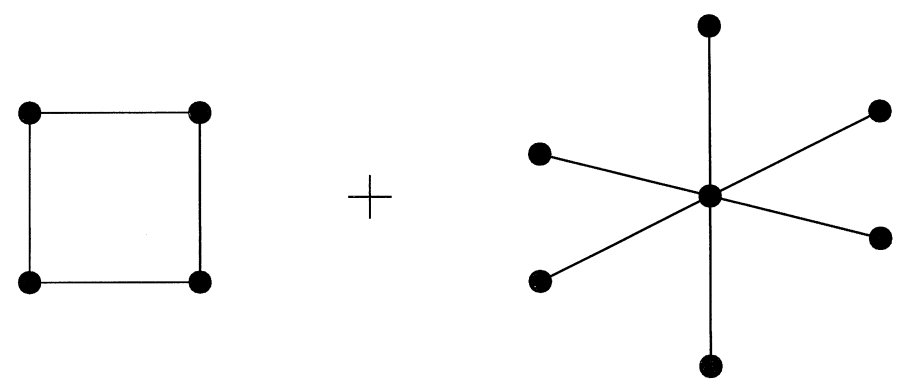

Figure 2. Symbolic representation of lattice equation and similarity constraint.

constraint (2.5) that couples the three lattice directions. Thus, for the three-dimensional case we have a coupled system of equations whose symbolic representation is shown in Figure 2.

In the previous section we have already demonstrated the consistency of the three copies of the lattice equation (2.2) amongst themselves. The framework given in [3] ensures the compatibility of the lattice equation and the similarity constraint in general. What remains to be shown in this first truly multidimensional case $N=3$ is how in a consistent way an initial value problem (IVP) can be formulated, and to demonstrate that this IVP is well-posed; i.e. that given a suitable choice of initial data, we are able to iterate the solution through the whole lattice and that the corresponding solution is single-valued at each lattice site.

In Figure 3 we have indicated how the iteration of the system proceeds. From a given configuration of initial data (located at the vertices indicated by $\bullet$ ) and moving through the lattice by calculating each point by means of either the lattice equation (points indicated by $\circ$ ) or the similarity constraint (points indicated by $\times$ ). The first point where a possible conflict arises, due to the fact that the corresponding values of the dependent variable can be calculated in more than one way, is indicated by $\otimes$. It is at such points that the consistency of the similarity reduction needs to be verified by explicit computation. This has been carried out for this three-dimensional

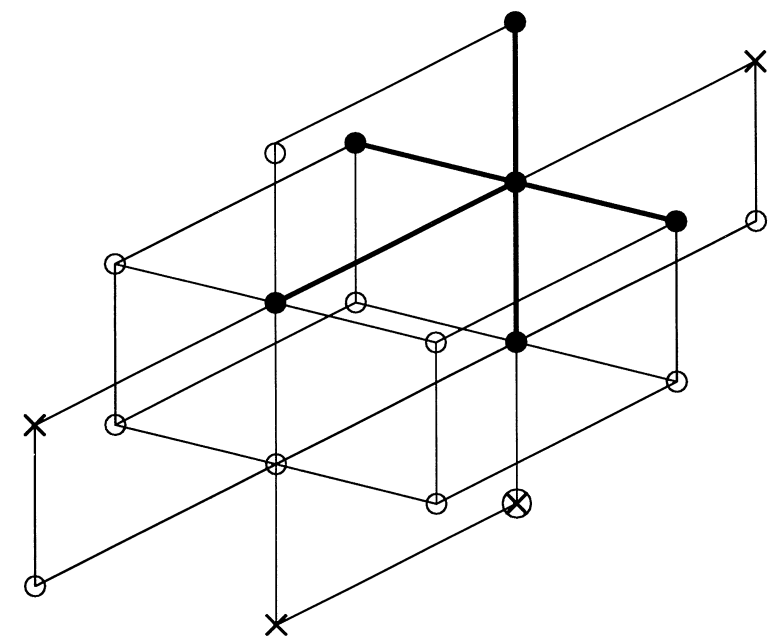

Figure 3. Consistency of the constrained lattice system. 
case using MAPLE. Obviously, the iteration involves too many steps and the expressions soon become too large to reproduce here. objects

In order to analyse the explicit reduction in this case, we redefine the following

$$
\begin{gathered}
a_{1}=a, a_{2}=b, a_{3}=c \\
X_{12}=X, X_{13}=Y, \mathcal{X}_{12}=\mathcal{X}, \mathcal{X}_{13}=\mathcal{Y}
\end{gathered}
$$

using also $n_{1}=n, n_{2}=m, n_{3}=h$, as well as $p_{1}=p, p_{2}=q$ and $p_{3}=r$ to simplify the notation. To start with the continuous equations, fixing the independent variable to be $p$ we obtain the following linear system for the quantities $b$ and $c$ from equation (2.14)

$$
\begin{aligned}
& {\left[\begin{array}{cc}
2 \mathcal{X} & \mathcal{X}+\mathcal{Y} \\
\mathcal{X}+\mathcal{Y} & 2 \mathcal{Y}
\end{array}\right]\left[\begin{array}{c}
m b \\
h c
\end{array}\right]=\left[\begin{array}{c}
\mu+v+p \frac{\partial}{\partial p} \log X \\
\mu+v+p \frac{\partial}{\partial p} \log Y
\end{array}\right]+} \\
& {\left[\begin{array}{c}
(\mu-v) \mathcal{X}+(n+m) \frac{p q}{p^{2}-q^{2}}\left(\frac{1}{X}-X\right)+h \frac{p r}{p^{2}-r^{2}}\left(\frac{1}{Y}-Y\right) \\
(\mu-v) \mathcal{Y}+(n+h) \frac{p r}{p^{2}-r^{2}}\left(\frac{1}{Y}-Y\right)+m \frac{p q}{p^{2}-q^{2}}\left(\frac{1}{X}-X\right)
\end{array}\right],}
\end{aligned}
$$

where we have used the similarity constraint to eliminate the quantity $a$. Furthermore, from (2.13) we obtain the differential relations

$$
\begin{aligned}
\frac{\partial(m b)}{\partial p}= & \frac{m q}{q^{2}-p^{2}}\left[(n+\mu-v-m b-h c)(1-b) \frac{1}{X}\right. \\
& -(1+b)(n-\mu+v+m b+h c) X], \\
\frac{\partial(h c)}{\partial p}= & \frac{h r}{r^{2}-p^{2}}\left[(n+\mu-v-m b-h c)(1-c) \frac{1}{Y}\right. \\
& -(1+c)(n-\mu+v+m b+h c) Y] .
\end{aligned}
$$

Solving $b$ and $c$ from the linear system (3.4), and substituting the results in the differential relations (3.5a) and (3.5b), we obtain a coupled system of second-order nonlinear ODE's of the form

$$
\begin{aligned}
& \frac{\partial^{2} X}{\partial p^{2}}=A_{1}\left(\frac{\partial X}{\partial p}\right)^{2}+A_{2}\left(\frac{\partial Y}{\partial p}\right)^{2}+A_{3}\left(\frac{\partial X}{\partial p}\right)\left(\frac{\partial Y}{\partial p}\right)+A_{4}\left(\frac{\partial X}{\partial p}\right)+A_{5}\left(\frac{\partial Y}{\partial p}\right)+A_{6}, \\
& \frac{\partial^{2} Y}{\partial p^{2}}=B_{1}\left(\frac{\partial X}{\partial p}\right)^{2}+B_{2}\left(\frac{\partial Y}{\partial p}\right)^{2}+B_{3}\left(\frac{\partial X}{\partial p}\right)\left(\frac{\partial Y}{\partial p}\right)+B_{4}\left(\frac{\partial X}{\partial p}\right)+B_{5}\left(\frac{\partial Y}{\partial p}\right)+B_{6},
\end{aligned}
$$

where each of the coefficients $A_{1} . . A_{6}, B_{1} . . B_{6}$ are functions of $X, Y, p$ and six free parameters; namely $\mu, v, n, m, h$ and $q / r$. These functions have been calculated explicitly using MAPLE but unfortunately are too large to reproduce here.

Alternatively, we can derive a system of second-order ordinary difference equations by fixing one of the discrete variables, say $n=n_{1}$, and using the relations (2.10) to obtain the equations 


$$
\begin{aligned}
& (p X-q) b+p X+q=\frac{\left(q^{2}-p^{2}\right) X}{q X-p}(\tilde{b}+1) \\
& (p Y-r) c+p Y+r=\frac{\left(r^{2}-p^{2}\right) Y}{r Y-p}(\tilde{c}+1),
\end{aligned}
$$

where the tilde denotes the shift in the lattice direction associated with the variable $n$. Using the similarity constraint

$$
n a+m b+h c=\mu-v, \quad v=\lambda(-1)^{n+m+h}
$$

to eliminate the variables $c$, we obtain the following linear system in terms of $\tilde{b}$ and $b$ :

$$
\begin{aligned}
& {\left[\begin{array}{cc}
\left(q^{2}-p^{2}\right) X & -(p X-q)(q X-p) \\
-m\left(r^{2}-p^{2}\right) Y & m(p Y-r)(r Y-p)
\end{array}\right]\left[\begin{array}{c}
\tilde{b} \\
b
\end{array}\right]=} \\
& {\left[\begin{array}{c}
(p X+q)(q X-p)-\left(q^{2}-p^{2}\right) X \\
(r Y-p)((p Y-r)(\mu-v-n a)+h(p Y+r))-\left(r^{2}-p^{2}\right) Y(h+\mu+v-(n+1) \tilde{a})
\end{array}\right]}
\end{aligned}
$$

where the $a$ and $\tilde{a}$ can be expressed in terms of $X$ and $Y$ by

$$
a=\frac{q X \underset{\sim}{X}+p(\underset{\sim}{X}-X)-q}{q X \underset{\sim}{X}-p(\underset{\sim}{X}+X)+q}=\frac{r Y \underset{\sim}{Y}+p(\underset{\sim}{Y}-Y)-r}{r Y \underset{\sim}{Y}-p+Y)+r},
$$

(where the undertilde denotes the backward shift with respect to the discrete variable). The system of equations (3.6), (3.7) and (3.9) — or, equivalently, (3.8) together with (3.9) leads effectively to a fourth-order ordinary difference equation in one variable. In fact, solving $b$ and $\tilde{b}$ from (3.8) and then eliminating $b$ altogether by a shift in the independent variable $n$ we get a coupled system containing one equation in terms of $X, \tilde{X}, \tilde{\tilde{X}}, \underset{X}{ }$ and $Y$, and the equation (3.9) which is first order in both $X$ and $Y$ with respect to the shift in the variable $n$. This system of equations depends on six free parameters, namely $\mu, v, m, h, q / p$ and $r / p$ and is quadratic in $Y$; hence we cannot explicitly reduce it to a single fourth-order ordinary difference equation in terms of $X$ and $n$ without evoking algebraic expressions. However it can be shown that the IVP for the coupled system of equations (3.8) and (3.9) is well posed; i.e. given initial data points $\underset{\sim}{X}, X, \tilde{X}$ and $Y$ at a fixed value of the discrete variable $n$, this system allows the subsequent iterates $\tilde{\tilde{X}}$ and $\tilde{Y}$ to be calculated uniquely by solving linear equations only.

4. Isomonodromic deformation problem. The isomonodromic deformation problem for the multidimensional lattice system is of Schlesinger type [22]. In the two-dimensional case it was already presented in [4] for special values of the parameters $\mu, v$; see also [3] for the general parameter case. The extension from the twodimensional to the multidimensional lattice is immediate: one only needs to introduce additional terms of similar form for each additional lattice direction. Thus, the Lax representation consists on the one hand of the linear shifts on the lattice of the form 


$$
\psi^{i}(\kappa)=T_{i} \psi(\kappa)=L_{i}(\kappa) \psi(\kappa),
$$

in which $\kappa$ is a spectral parameter, and where the Lax matrices $L_{i}$ are given by

$$
L_{i}(\kappa)=\left(\begin{array}{cc}
p_{i} & v^{i} \\
\frac{\kappa}{v} & p_{i} \frac{v^{i}}{v}
\end{array}\right)
$$

leading to the Lax equations

$$
L_{i}^{j} L_{j}=L_{j}^{i} L_{i}
$$

which lead to a copy of the lattice MKdV equation on each two-dimensional sublattice labelled by the indices $(i, j)$. On the other hand we have the linear differential equation for $\psi(\kappa)$ with respect to its dependence on the spectral variable $\kappa$ :

$$
\begin{aligned}
\kappa \frac{d}{d \kappa} \psi(\kappa) & =\frac{1}{2}\left(\begin{array}{cc}
-(1+\mu) & 0 \\
0 & \lambda(-1)^{\sum_{i} n_{i}}+\sum_{i} n_{i}
\end{array}\right) \psi(\kappa) \\
& +\sum_{i} \frac{n_{i} v}{v^{i}+{ }_{i} v}\left(\begin{array}{cc}
0 & v^{i} \\
0 & -p_{i}
\end{array}\right) T_{i}^{-1} \psi(\kappa),
\end{aligned}
$$

the compatibility of which with (4.1) leads to the similarity constraint (2.5). In addition, we have differential equations for $\psi$ in terms of its dependence on the lattice parameters $p_{i}$ which are of the form

$$
\frac{\partial \psi}{\partial p_{i}}=\frac{n_{i}}{p_{i}}\left(\begin{array}{cc}
1 & 0 \\
0 & 0
\end{array}\right) \psi+\frac{2 n_{i} v}{v^{i}+{ }_{i} v}\left(\begin{array}{cc}
0 & -\frac{1}{p_{i}} v^{i} \\
0 & 1
\end{array}\right) T_{i}^{-1} \psi,
$$

for each of the variable $p_{i}$. It is the variables $t_{i}=p_{i}^{2}$ that play the role as independent variables in the continuous $\mathrm{P}_{\mathrm{VI}}$ hierarchy.

The elimination of the back-shifted vectors $T_{i}^{-1} \psi$ by using the inverse of the Lax relations (4.1) leads to the following linear differential equation for $\psi$ :

$$
\frac{\partial \psi}{\partial \kappa}=\left(\frac{A_{0}}{\kappa}+\sum_{i} \frac{A_{i}}{\kappa-t_{i}}\right) \psi
$$

thus leading to the problem in the Schlesinger form, with regular singularities at $0, \infty,\left\{t_{i}\right\}$. The matrices $A_{0}$ and $A_{i}$ are given by

$$
\begin{aligned}
& A_{0}=\frac{1}{2}\left(\begin{array}{cc}
-(\mu+1) & \sum_{i} \frac{n_{i}}{p_{i}}\left(1-a_{i}\right) v^{i} \\
0 & \lambda \sum_{i} n_{i}+\sum_{i} n_{i} a_{i}
\end{array}\right), \\
& A_{i}=n_{i}\left(\begin{array}{cc}
\frac{1}{2}\left(1+a_{i}\right) & -\frac{1}{2 p_{i}} v^{i}\left(1-a_{i}\right) \\
-\frac{p_{i}}{2 v^{i}}\left(1+a_{i}\right) & \frac{1}{2}\left(1-a_{i}\right)
\end{array}\right) .
\end{aligned}
$$

The continuous isomonodromic deformation is provided by the linear differential equations in terms of the lattice parameters; namely 


$$
\frac{\partial \psi}{\partial t_{i}}=\left(P_{i}-\frac{A_{i}}{\kappa-t_{i}}\right) \psi
$$

where

$$
P_{i}=\frac{n_{i}}{2 p_{i}}\left(\begin{array}{cc}
-\frac{1}{p_{i}} a_{i} & 0 \\
\frac{1}{v^{i}}\left(1+a_{i}\right) & 0
\end{array}\right) .
$$

Equation (4.7) is not quite in standard form, and we need to apply a gauge transformation of the form

$$
\bar{\psi} \equiv V \psi, \quad V=\left(\begin{array}{cc}
1 / v & 0 \\
U / v & 1
\end{array}\right),
$$

to remove the term with $P_{i}$, where the auxiliary variable $U$ obeys an interesting set of equations by itself; (in fact this is the object obeying the lattice $\mathrm{KdV}$ system of equations); cf. [3], but we shall not give any details here. With this gauge, the continuous isomonodromic deformation (4.7) becomes the standard form

$$
\frac{\partial \bar{\psi}}{\partial t_{i}}=-\frac{\bar{A}_{i}}{\kappa-t_{i}} \bar{\psi}, \quad \bar{A}_{i}=V A_{i} V^{-1},
$$

whilst the discrete isomonodromic condition is readily obtained from the Lax representation of (4.1).

5. Connection with Garnier systems. Interestingly, already M. R. Garnier in his seminal paper [15] of 1912, embarked on the question of finding higher-order analogues of the PVI equation, adopting the method that was proposed somewhat earlier by R. Fuchs, in [16], that can be identified with the isomonodromic deformation approach; see also [22]. Garnier gave a general construction of such higher-order equations constituting coupled systems of partial differential equations, which are the isomonodromic Garnier systems. As a particular example, he wrote down explicitly in [15] the first higher-order $\mathrm{P}_{\mathrm{VI}}$ equation in terms of the following coupled system, consisting of the second-order ODE in terms of two dependent variables $w=w(t, s)$ and $z=z(t, s)$

$$
\begin{aligned}
\frac{\partial^{2} w}{\partial t^{2}} & =\frac{1}{2}\left(\frac{1}{w}+\frac{1}{w-1}+\frac{1}{w-t}+\frac{1}{w-s}-\frac{1}{w-z}\right)\left(\frac{\partial w}{\partial t}\right)^{2} \\
& -\left(\frac{1}{t}+\frac{1}{t-1}+\frac{1}{t-s}-\frac{1}{t-w}-\frac{1}{t-z}\right) \frac{\partial w}{\partial t} \\
& +\frac{1}{2} \frac{w(w-1)(w-s)(z-t)}{z(z-1)(z-s)(w-t)(z-w)}\left(\frac{\partial z}{\partial t}\right)^{2}-\frac{w-t}{(z-t)(z-w)}\left(\frac{\partial w}{\partial t}\right)\left(\frac{\partial z}{\partial t}\right) \\
& +\frac{2 w(w-1)(w-t)(w-s)(z-t)^{2}}{t^{2}(t-1)^{2}(t-s)^{2}(w-z)} \times \\
& \times\left[\alpha+\beta+\gamma+\delta+\kappa+\frac{7}{4}-\frac{t s}{z} \frac{\alpha+\frac{1}{4}}{w^{2}}+\frac{(t-1)(s-1)}{(z-1)} \frac{\beta+\frac{1}{4}}{(w-1)^{2}}\right. \\
& \left.+\frac{t(t-1)(t-s)}{(z-t)} \frac{\gamma}{(w-t)^{2}}+\frac{s(s-1)(s-t)}{(z-s)} \frac{\delta}{(w-s)^{2}}\right]
\end{aligned}
$$


together with coupled first order PDE's

$$
\begin{gathered}
\frac{t(t-1)}{t-z} \frac{\partial w}{\partial t}+\frac{s(s-1)}{s-z} \frac{\partial w}{\partial s}=\frac{w(w-1)}{w-z} \\
\frac{t(t-1)}{t-w} \frac{\partial z}{\partial t}+\frac{s(s-1)}{s-w} \frac{\partial z}{\partial s}=\frac{z(z-1)}{z-w} .
\end{gathered}
$$

It should be pointed out that the system consisting of (5.1a), (5.1b) and (5.1c) amounts actually to a fourth order ODE in terms of $w=w(t)$ only, and as such can be rightly considered to be the first higher-order member of the Painlevé VI hierarchy. In fact, Garnier gave in his paper a number of important assertions: (i) that his system of equations is completely integrable ${ }^{1}$, and that it degenerates (under the autonomous limit) to a system living on the Jacobian of a hypereliptic curve, (ii) that the symmetric combinations of the dependent variables of the system, as functions of each one of the essential singularities (i.e. singling out one of the independent variables) are meromorphic in terms of this variable except for the fixed critical points which are at $0,1, \infty$, or at the location of the values of the other independent variables $^{2}$; (iii) that for the parameters of the system in general position the symmetric functions of the dependent variables are essentially transcendental functions of the constants of integration (i.e. of the initial data).

Subsequent work on the Garnier systems was done mostly by K. Okamoto and his school; see, for example $[\mathbf{2 3}, \mathbf{2 4}]$. However, it seems that in most of these works these systems were treated rather as an overdetermined system of PDE's rather than (as Garnier himself clearly had in mind) as a consistent system of ODE's. Although it is not easy to find the explicit transformation of the lattice system exposed in Sections 2 and 3 to the systems that Garnier wrote down, in particular to find the explicit relation between the above system (5.1) and the system consisting of (3.4) and (3.5), it is to be expected that such a mapping exists. The identification is probably easiest to obtain via the transformation of the corresponding Schlesinger type of system as given in Section 4 and the linear system that Garnier exploited in [15]. However, the search for such an identification will be left to a future study.

Let us finish with some remarks on the relevance of these results for work that has been done in recent years. One of the most exciting developments is the way in which the issue of algebraic solutions of $\mathrm{P}_{\mathrm{VI}}$ have arisen in recent years; e.g. in connection with WDVV equations, Frobenius manifolds and quantum cohomology; see, for example the review [25]. Such algebraic solutions were already known to Picard, Painlevé and Chazy. In fact, in his early paper [16], R. Fuchs obtained a realisation of $\mathrm{P}_{\mathrm{VI}}$ in terms of an elliptic integral, and this realisation was subsequently used by Painleve in [26] to derive an elliptic form for the $\mathrm{P}_{\mathrm{VI}}$ equation, a form of the equation that was recently recovered by Manin in $[\mathbf{1 8}]^{3}$. The assertions of Garnier in [15] on his generalisation of the Fuchs' approach might form a starting point for extending this elliptic connection to the Garnier systems, in which case we should expect to be able to find a realisation of those systems in terms of hyperelliptic integrals rather than elliptic ones. This might eventually lead to the

\footnotetext{
${ }^{1}$ Obviously, Garnier's use of the term integrability was meant here in the precise sense of that of a compatible system, very much in the same sense as the compatibility of the continuous and discrete systems that we have encountered in Sections 2 and 3.

${ }^{2}$ This assertion amounts to the well-known Panlevé property.

${ }^{3}$ We are grateful to $\mathrm{R}$. Conte for pointing out reference [26].
} 
construction of algebraic solutions of those systems, possibly in the spirit of the recent papers $[\mathbf{2 7 , 2 8}]$. It would be of interest to further investigate the role of the discrete systems in connection with the Garnier systems: we expect them to constitute the superposition formulae for the underlying higher root systems of the corresponding affine Weyl groups. Thus, eventually, a geometric interpretation of the Garnier systems and their discrete analogues in the sense of the blowings-up of the corresponding rational surfaces of their initial conditions, along the lines of the recent paper [2], might be anticipated.

\section{REFERENCES}

1. B. Grammaticos, F.W. Nijhoff and A. Ramani, Discrete Painlevé equations, in The Painlevé property, one century later, edited by R. Conte (Springer-Verlag, 1999), 413-516.

2. H. Sakai, Rational surfaces associated with affine root systems and geometry of the Painlevé equations, Preprint, (Kyoto University, May 1999).

3. F. W. Nijhoff, A. Ramani, B. Grammaticos and Y. Ohta, On discrete Painlevé equations associated with the lattice KdV systems and the Painlevé VI equation, solv-int/ 9812011, Stud. Appl. Math., to appear.

4. F. W. Nijhoff and V. G. Papageorgiou, Similarity reductions of integrable lattices and discrete analogues of the Painlevé II equation, Phys. Lett. A 153 (1991), 337-344.

5. F. W. Nijhoff, Discrete Painlevé equations and symmetry reduction on the lattice, in Discrete integrable geometry and physics, edited by A. I. Bobenko and R. Seiler (Oxford University Press, to appear).

6. F. W. Nijhoff, N. Joshi and A. Hone, On the discrete and continuous Miura chain associated with the sixth Painlevé equation, Phys. Lett. A 267 (2000), 147-156.

7. F. W. Nijhoff, A. Hone and N. Joshi, On a Schwarzian PDE associated with the KdV hierarchy, Phys. Lett. A 264 (2000), 396-406.

8. H. Flaschka and A. C. Newell, Monodromy- and spectrum-preserving deformations I, Comm. Math. Phys. 76 (1980), 65-116.

9. N. A. Kudryashov, The first and second Painlevé equations of higher order and some relations between them, Phys. Lett. A 224 (1997), 353-360.

10. C. Creswell and N. Joshi, The discrete Painlevé I hierarchy, in Symmetries and integrability of difference equations, edited by P. A. Clarkson and F. W. Nijhoff (Cambridge University Press, 1999), 197-205.

11. A. N. W. Hone, Non-autonomous Hénon-Heiles systems, Phys. D 118 (1998), 1-16.

12. M. Noumi and Y. Yamada, Higher order Painlevé equations of type $A_{l}^{(1)}$, Funkcial. Ekvac. 41 (1998), 483-503.

13. P. A. Clarkson, N. Joshi and A. Pickering, Bäcklund transformations for the second Painlevé hierarchy: a modified truncation approach, Inverse Problems 15 (1999), 175-187.

14. A. V. Kitaev, Elliptic asymptotics of the first and the second Painleve transcendents, Russian Math, Surv. 49 (1994), 81-150.

15. R. Garnier, Sur des équations différentielles du troisieme ordre dont l'intégrale générale est uniforme et sur une classe d'équations nouvelles d'ordre supérieur, Ann. Écol. Norm. Sup., 29 (1912), 1-126.

16. R. Fuchs, Sur quelques équations differentielles lineaires du second ordre, $C$. $R$. Acad. Sci. (Paris) 141 (1905), 555-558. Math. Ann. 63 (1907), 301-321.

17. N. J. Hitchin, Poncelet polygons and the Painleve transcendents, in Geometry and analysis, edited by Ramanan, (Oxford University Press, 1995), 151-185.

18. Yu. I. Manin, Sixth Painlevé equation, universal elliptic curve and mirror of $\mathbb{P}^{2}$, alggeom/9605010.

19. A. M. Levin and M. A. Olshanetsky, Painlevé-Calogero correspondence, ITEPTH23/97, alg-geom/9706010, preprint.

20. B. Dubrovin and M. Mazzocco, Monodromy of certain Painlevé VI transcendents and reflection groups, math.AG/9806056.

21. F. W. Nijhoff and H. W. Capel, The discrete Korteweg-de Vries equation, Acta Appl. Math. 39 (1995), 133-158. 
22. L. Schlesinger, Über eine Klasse von Differentialsystemen beliebiger Ordnung mit festen kritischen Punkten, J. für Math. 141 (1912), 96-145.

23. K. Okamoto, Isomonodromic deformation and Painlevé equations, and the Garnier system, J. Fac. Sci. Univ. Tokyo Sect. IA Math., 33 (1986), 575-618.

24. K. Iwasaki, H. Kimura, S. Shimomura and M. Yoshida, From Gauss to Painlevé. A modern theory of special functions (Vieweg-Verlag, Braunschweig, 1991), Ch. 3.

25. B. Dubrovin, Painlevé transcendents in two-dimensional topological field theory, in The Painlevé property, one century later, edited by R. Conte, (Springer-Verlag, 1999), 287-412.

26. P. Painlevé, Sur les équations différentielles du second ordre à points critiques fixes, C.R. Acad. Sci. (Paris) 143 \#26 (1906), 1111-1117.

27. A. V. Kitaev and D. A. Korotkin, On solutions of the Schlesinger equations in terms of $\Theta$-functions, math-ph/9810007.

28. P. Deift, A. Its, A. Kapaev and X. Zhou, On the algebra-geometric integration of the Schlesinger equations, (Courant Institute, 1999), preprint. 